\title{
Study on thermal oxidation and combustion of aluminum ultrafine powder in high-energy material
}

\author{
Alexander G. Korotkikh ${ }^{1,2, *}$, Konstantin V. Slyusarskiy ${ }^{1}$, Konstantin A. Monogarov ${ }^{1}$, and \\ Ekaterina A. Selikhova ${ }^{1}$ \\ ${ }^{1}$ National Research Tomsk Polytechnic University, 634050 Tomsk, Russia \\ ${ }^{2}$ Tomsk State University, 634050 Tomsk, Russia
}

\begin{abstract}
Aluminum is a promising metal fuel for solid propellants due to the high heat of combustion and propulsion specific impulse values. In current study the results of aluminum-containing high-energy material oxidation and combustion processes were received by means of the thermogravimetry and a constant pressure bomb. Ammonium perchloratebased high-energy materials with aluminium powder with different particles size and genesis (produced by methods of electrical explosion of wire is ultrafine Alex and spraying is micron ASD) were studied. The oxidation process mechanism and activation energy were obtained using Freidman and Kissinger methods. It was found that the average activation energy values for tested HEM samples were in the range of 120 $160 \mathrm{~kJ} / \mathrm{mole}$ and the burning rate for HEM sample with Alex was 2.53.0 times higher than HEM sample with ASD-1.
\end{abstract}

\section{Introduction}

Last 15 years the intense research on new generation of solid and gel-like high energetic systems with aluminium powders of different dispersity [1, 2]. These research is coordinated array of fundamental and applied studies in sphere of development of perspective solid propellants (SP) based on aluminum ultrafine powder (UFP) [3]. They are expected to allow to create and implement SP with energetic characteristics on principally new level. There are several methods of aluminum UFP production, two of them are the most widespread: method of pneumatic molten metal spraying and electrical explosion [3]. Obtained by these methods powders have different dispersity and particle form. This has significant influence on ignition and combustion process characteristics.

Simultaneous thermal analysis allows to obtain reliable data on oxidation process kinetics with high preciseness. Many presented in literature articles are devoted to analysis of oxidation kinetics in air medium of aluminium powder with different dispersity and genesis $[4,5]$. In majority of high energy materials (HEM) the metal powders are used together with solid oxidizer and binder. Combustion kinetics is significantly affected by oxidizer type too. There are few studies of this type in scientific literature and all of them

*Corresponding author: korotkikh@tpu.ru 
are limited by quite narrow range of HEMs like in [6], where kinetic analysis of HEM oxidation based on HTPB and aluminium nanopowder in air medium. For propulsion applications the HEM linear combustion rate is also important. To define linear combustion rate the bomb with constant pressure and method of burning wires were used as one of the most precise.

The combustion characteristics of HEM based on ammonium perchlorate and aluminium powders produced by electrical explosion and pneumatic spraying in argon medium via simultaneous thermal analysis and method of burning wires.

\section{Materials and methods}

\subsection{HEM samples}

The HEM samples content was two fractions of ammonium perchlorate (AP) (sieve fractions less than $50 \mu \mathrm{m}$ and $160-315 \mu \mathrm{m}$ with mass ratio 40/60), butadiene rubber plasticized by transformer oil (19.7 wt. \%), and aluminum powder (15.7 wt. \%). Metal fuel consists of 85-99 wt. \% of active aluminum depending on the powder dispersity. In tested HEM samples we used aluminum different grade: micron powders ASD-4 (HEM with this powder further in text would be referred as ASD-4), ASD-6 (ASD-6), ASD-10 (ASD-10) and obtained by electrical explosion of wire UFP Alex (Alex).

Characteristic sizes of aluminum powder particles were defined using experimental histograms from manufacturer. Physical-chemical properties defining revealed that poured density of produced by pneumatic method aluminum powder with dispersity $0.3-0.5 \mu \mathrm{m}$ is $0.54 \mathrm{~g} / \mathrm{sm}^{3}$. It is 3.6 times higher compared to UFP Alex and 2.0 times lower than ASD-4 powder. This difference may be connected to particle structure and oxide layer thickness. According to images of electron X-ray microscope and X-ray diffraction analysis of Alex UFP with particle size $0.1 \mu \mathrm{m}$ its oxide layer has poured structure and with gas content up to 3 wt. \%. Dispersed composition of aluminum powders is given in Table 1.

Table 1. The characteristic diameters of aluminum powder particles.

\begin{tabular}{|c|c|c|c|c|c|}
\hline Aluminum powder & $d_{10}, \mu \mathrm{m}$ & $d_{20}, \mu \mathrm{m}$ & $d_{30}, \mu \mathrm{m}$ & $d_{32}, \mu \mathrm{m}$ & $d_{43}, \mu \mathrm{m}$ \\
\hline ASD-4 & 1.23 & 1.66 & 2.28 & 4.34 & 7.34 \\
\hline ASD-6 & 0.85 & 1.17 & 1.60 & 3.01 & 4.72 \\
\hline Alex & 0.12 & 0.13 & 0.14 & 0.16 & 0.18 \\
\hline
\end{tabular}

\subsection{Thermal analysis}

Thermal analysis of samples was carried out via simultaneous TG/DSC/DTA analyzer Netzsch STA 449 F3 Jupiter in the temperature range of $30-1000{ }^{\circ} \mathrm{C}$ with three heating rates: 2,4 and $10{ }^{\circ} \mathrm{C} / \mathrm{min}$. Measurements were made with hinge mass $2.5 \mathrm{mg}$ in argon medium with the volumetric flow rate of $10 \mathrm{ml} / \mathrm{min}$. Before each measurement the chamber of analyzer was blown with argon to eliminate remaining air.

\subsection{Kinetic analysis}

Kinetic analysis of HEM oxidation process was realized according to presented in [7] methodic using isoconversional algorithms of Friedman and Kissinger-Akahira-Sunose.

Differential method of Friedman is based on following equation: 


$$
\ln \left(i \frac{d \alpha}{d T}\right)_{\alpha, i}=\ln \left[f(\alpha) A_{\alpha}\right]-\frac{E_{\alpha}}{R T_{\alpha, i}}
$$

where $\alpha$ is the extent of conversion; $f(\alpha)$ is a kinetic function; $A_{\alpha}$ is the frequency factor, wt. $\% / \mathrm{s} ; E$ is the activation energy; $\mathrm{J} /$ mole; $R$ is the universal gas constant, $\mathrm{J} /($ mole $\mathrm{K}) ; T$ is the temperature, $\mathrm{K} ; i$ is the heating rate, $\mathrm{K} / \mathrm{min}$.

The activation energy was defined using approximation curve slope at fixed conversion for each heating rate.

Integral method of Kissinger-Akahira-Sunose is based on following approach to solution of temperature integral:

$$
\ln \left(\frac{i}{T_{\alpha, i}^{2}}\right)=\text { Const }-\frac{E_{\alpha}}{R T_{\alpha, i}} .
$$

Simultaneous application of both integral and differential methods allows to decrease negative influence of each separate method.

\subsection{Constant pressure bomb}

The burning rate experiments were carried out using bomb of constant pressure via method of burning wires in argon in the nitrogen pressure range of 1-8 MPa. Cylindrical samples with diameter $10 \mathrm{~mm}$ and height $30 \mathrm{~mm}$ with fortified side surface. The used aluminum powders content was changed from 0 to $20 \mathrm{wt}$. $\%$.

\section{Results and discussion}

\subsection{Thermal analysis}

Thermal analysis data for tested HEM samples were obtained. Form of TG and DTG curves is given in Fig.1 on example of HEM sample with Alex.

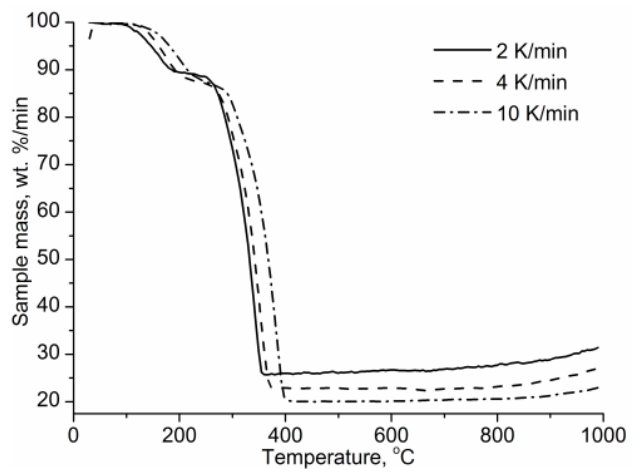

a)

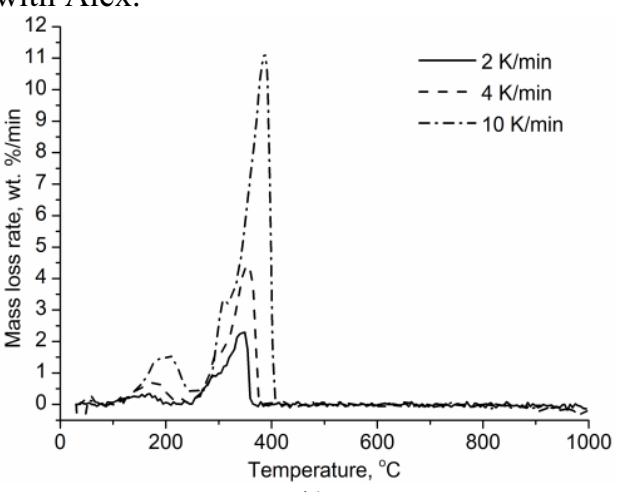

b)

Fig. 1. TG (a) and DTG (b) curves for tested HEM with Alex.

Form of DTG-curves shows that HEM thermal decomposition and aluminum oxidation has two stages. The first stage takes place in the temperature range of $100-250{ }^{\circ} \mathrm{C}$ and connected to primary decomposition of oxidizer and binder. Second stage takes place in the temperature range of $250-400{ }^{\circ} \mathrm{C}$ and caused by aluminum oxidation with high-temperature 
decomposition of oxidizer and binder. Additional peak during the second stage at temperatures close to $300{ }^{\circ} \mathrm{C}$ may be connected to further decomposition of ammonium perchlorate.

Experimental dependences of extent of conversion for tested HEM samples vs. the temperature at two heating rates values are given in Fig. 2.

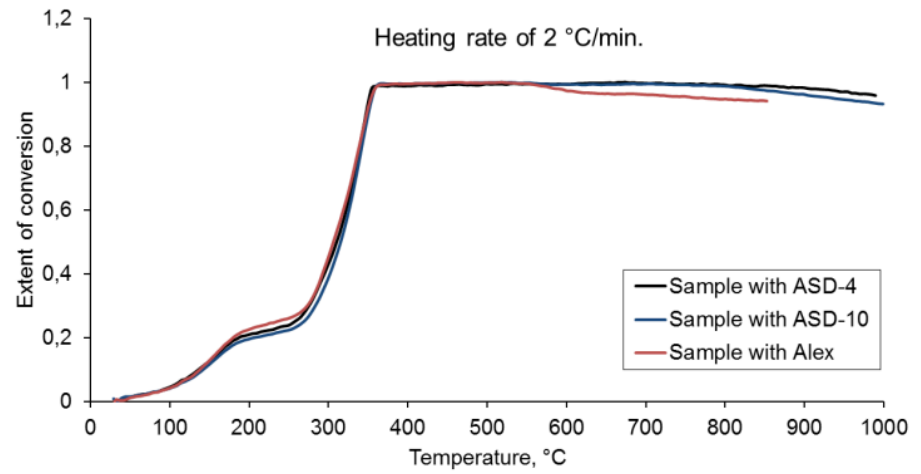

a)

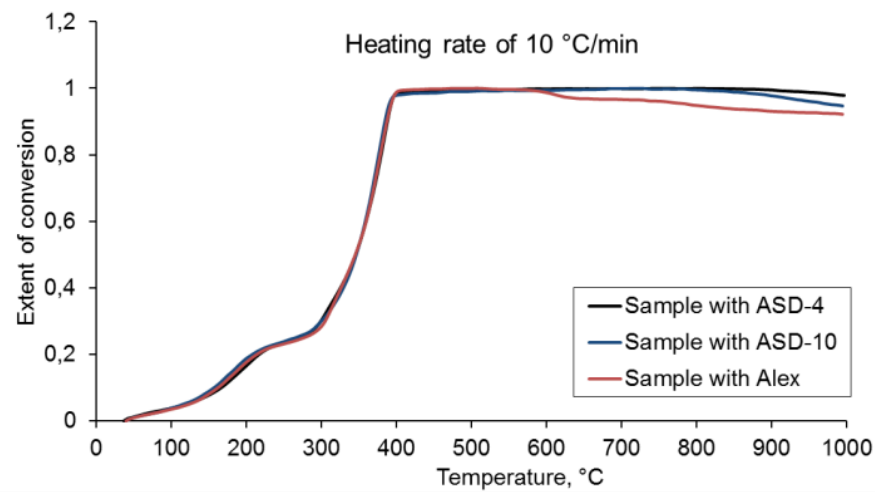

b)

Fig. 2. The HEM samples extent of conversion vs. temperature at heating rate of $2 \mathrm{~K} / \mathrm{min}$ (a) and 10 $\mathrm{K} / \mathrm{min}(\mathrm{b})$.

Fig. 2 illustrates two-staged character of HEM oxidation process and it does not changes with heating rate. At the same time the curve position shifts into area of higher temperatures at $30^{\circ} \mathrm{C}$. It results into moving of the first peak from 170 to $200{ }^{\circ} \mathrm{C}$ and the second peak from 360 to $390{ }^{\circ} \mathrm{C}$.

Comparison of extent of conversion vs temperature graphs for all samples reveals that at $10 \mathrm{~K} / \mathrm{min}$ the differences between HEM with different powders are insignificant in the temperature range of $50-400{ }^{\circ} \mathrm{C}$. At $2{ }^{\circ} \mathrm{C} / \mathrm{min}$ the oxidation rate of Alex HEM sample is higher than for ASD-4 and ASD-10 HEM samples. At the same time the mass gain rate for Alex sample is much higher compared to other samples. It could be concluded that at close to equilibrium conditions of TG-chamber the kinetic of HEM oxidation does not depend on dispersed composition of metal powder.

\subsection{Activation energy}

The activation energy dependences of oxidation process for tested HEM samples were obtained using two methods (Friedman and Kissinger-Akahira-Sunose) and are presented in Fig. 3. 


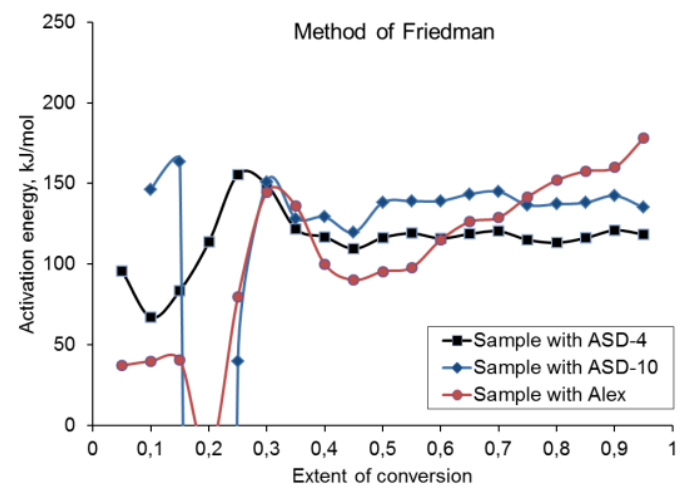

a)

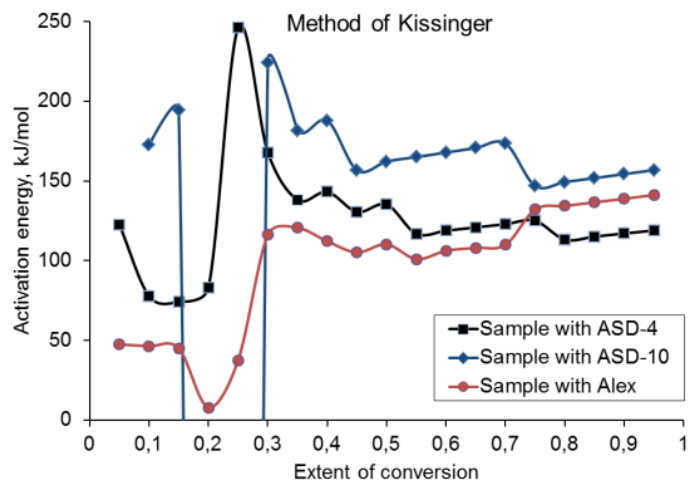

b)

Fig.3. The HEM activation energy vs. extent of conversion by Friedman (a) and KAS (b) methods.

While the activation energy for processes with several parallel reactions described by Friedman and KAS methods with significant error of the activation energy in the first stage varies in the wide range of values (from 0 to $250 \mathrm{~kJ} / \mathrm{mole}$ ) in the conversion range of $0.05-$ 0.35 where organic binding decomposition takes place. In the conversion range of $0.4-0.95$ the aluminum oxidation takes place and the activation energy values varies in more narrow range of $120-160 \mathrm{~kJ} / \mathrm{mole}$. These values are in good correspondence with literature data [8]. The average activation energy values during HEM oxidation are presented in Table 2.

Table 2. The average activation energy for HEM oxidation process.

\begin{tabular}{|c|c|c|c|}
\hline \multirow{2}{*}{ Calculation method } & \multicolumn{3}{|c|}{ HEM sample } \\
\cline { 2 - 4 } & Alex & $\begin{array}{c}\text { ASD- } \\
4\end{array}$ & $\begin{array}{c}\text { ASD- } \\
10\end{array}$ \\
\hline Friedman & 129 & 117 & 137 \\
\hline KAS & 120 & 123 & 162 \\
\hline
\end{tabular}

In the conversion range of $0.05-0.60$ the activation energy of HEM with Alex is lower compared to other samples. At the higher conversion values the activation energy of HEM with Alex increases. It may be caused by interference of secondary aluminum oxidation that is less intense compared to primary oxidation.

\subsection{Burning rate}

The burning rate values and combustion lows for HEM samples are presented in Fig. 4. 


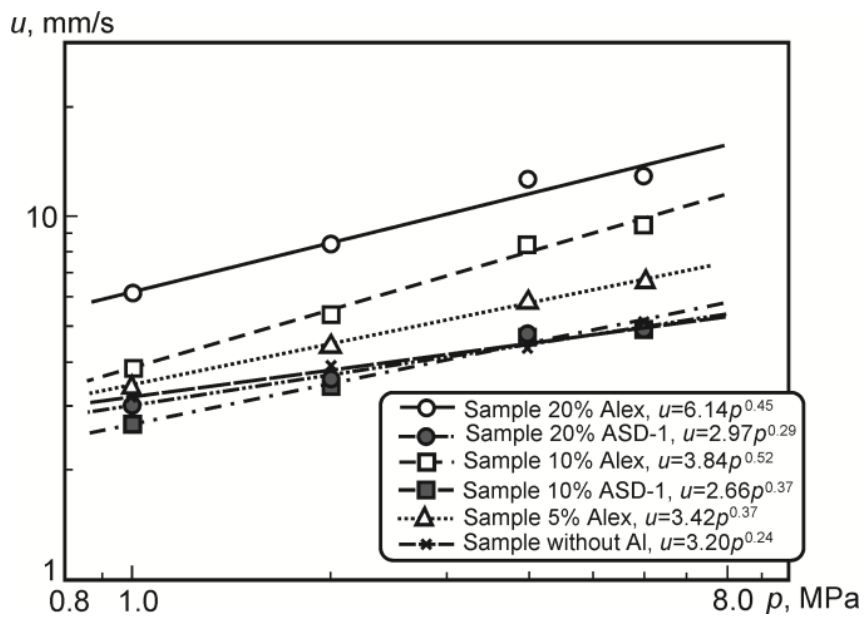

Fig. 4. The burning rate for tested HEM samples with aluminum powder.

The burning rate for tested HEM samples increases with growth of pressure in bomb. The burning rate for HEM sample with ASD-1 powder does not depend on metal content while for HEM with Alex this dependence is linear - lowering of metal content in HEM causes decreasing of the burning rate. It could be seen, that the burning rate for HEM samples with 20 wt. \% of Alex UFP is 2.5-3.0 times higher than for HEM with ASD-1 micron powder. With decrease in the metal content in HEM sample this difference decreases as well.

Influence of aluminium powder particle size on HEM combustion rate may be described by thermal theory of combustion and particle oxidation mechanism. If aluminium powder particles quickly ignite and burn near HEM sample surface than overall combustion rate of HEM will increase. To reach it the particle size should be rather small while temperature and composition of combustion products should be advantageous for aluminium particles ignition and combustion. Increasing of combustion rate with addition of aluminium UFP into HEM is connected to increasing of chemical reaction rate due to higher temperature in gas phase.

\section{Conclusion}

The study on thermal decomposition, oxidation and combustion for high-energy materials based on ammonium perchlorate, butadiene rubber and aluminium ultrafine or micron powders is presented. Thermal analysis data for tested HEM samples were obtained via Netzsch STA 449 F3 Jupiter analyser at the heating rates of 2,4 and $10{ }^{\circ} \mathrm{C} / \mathrm{min}$ in argon. The combustion experiment was carried out using the bomb of constant pressure via method of burning wires.

The TG and DTG-curves for HEM oxidation process were obtained as well as the activation energy dependences on extent of conversion. The HEM activation energy value varies in the range of $0-250 \mathrm{~kJ} / \mathrm{mole}$ at the $0.05-0.35$ extent of conversion and in the range of $120-160 \mathrm{~kJ} / \mathrm{mole}$ at the $0.40-0.95$ extent of conversion. The mass loss rate and the activation energy values are close to each other for HEM samples with aluminium powder of different dispersity.

The burning rate tends to increase at growth of ambient pressure and Alex UFP content in HEM sample while the burning rate for sample with ASD-1 micron powder does not change with variation of metal content and close to values of HEM without metal. At equal conditions the burning rate of HEM sample with $20 \mathrm{wt}$. \% Alex are 2.5-3.0 times higher 
than it with 20 wt. \% ASD-1. With decrease of aluminum content the burning rate difference between HEM samples with Alex and ASD powders decreases. It reveals that in unsteady conditions the dispesity and content of aluminum fuel in HEM play important role in the combustion process.

The reported study was supported by Russian Scientific Foundation, Grant 16-19-10316.

\section{References}

1. V.A. Arkhipov, A.G. Korotkikh, V.T. Kuznetsov, A.A. Razdobreev, I.A. Evseenko, Russ. J. Phys. Chem. B, 5, 616 (2011)

2. F. Maggi, A. Bandera, L. Galfetti, L.T. De Luca, T.L. Jackson, Acta Astranaut, 66, 1563 (2010)

3. Y. Li, C. Hu, Z. Deng, C. Li, H. Sun, Y. Cai, Acta Astranaut, 133, 455 (2017)

4. A.A. Il'in, R.N. Rumyantsev, V.V. Veisgaim, A.P. Il'in, Russ. J. Phys. Chem. A, 90, 764 (2016)

5. A.B. Vorozhtsov, M. Lerner, N. Rodkevich, H. Nie, A. Abraham, M. Schoenitz, E.L. Dreizin, Thermochim. Acta, 636, 48 (2016)

6. X. Lu, M. Zha, Z. Ma, F. Zhao, S. Xu, H. Xu, Combust. Sci. Technol. 189, 312 (2016)

7. S. Vyazovkin, K. Chrissafis, M.L. Di Lorenzo, N. Koga, M. Pijolat, B. Roduit, N. Sbirrazzuoli, J.J. Suñol, Thermochim. Acta, 590, 1 (2014)

8. Q.S.M. Kwok, R.C. Fouchard, A-M. Turcotte, P.D. Lightfoot, R. Bowesand, D.E.G. Jones, Propellants Explos. Pyrotech. 27, 229 (2002) 